\title{
Non-planar motions of a string vibrating against a smooth unilateral obstacle
}

\author{
Harkirat Singh ${ }^{1, a}$, Ashok K. Mandal ${ }^{1}$, and Pankaj Wahi ${ }^{1}$ \\ Indian Institute of Technology, Kanpur-208016, India
}

\begin{abstract}
Earlier theoretical studies on string vibrations against smooth unilateral obstacle assumed planar motions. The present paper analyzes the non planar motions of a string vibrating against a smooth unilateral curved obstacle. In particular, we study the effect of obstacle in determining different types of non planar motions of a string. The tension is assumed to be variable along the length due to the stretching of the string, which introduces nonlinear coupling between the perpendicular modes. The system of equations has been discretized by assuming functional form of the modes which satisfies all the geometrical boundary conditions. The mathematical model has been numerically investigated for different initial conditions and chosen value of obstacle parameters. The trajectory of a typical point on the string is oscillating ellipse and it transforms into rotating ellipses with time varying major and minor axis for larger amplitudes.
\end{abstract}

\section{Introduction}

Strings are the simplest continuous systems which exhibit vibrations. The applications of strings are quite diverse, for example, in cranes to lift loads and transmit power, in ropeways to suspend heavy weights, in elevators as a support system, in a classical Atwood machine as a classroom demonstration to verify laws of motion, etc. At high school level, the physics behind all these exercises is conceived by treating the string to be inextensible and massless entity. However, in reality the string deforms as well as possess certain mass density which immediately usher in the idea of vibrating strings. The mathematical model for small vibrations of a tightly stretched elastic string proposed by D'Alembert is given by

$$
\frac{\partial^{2} y}{\partial t^{2}}=c^{2} \frac{\partial^{2} y}{\partial x^{2}}, \quad c^{2}=\frac{T}{\rho},
$$

where "tightly stretched" implies that the tension $T$ is a constant, and $\rho$ denotes the constant mass density per unit length. Thus, dynamics of a string is ingested in a linear partial differential equation. Given initial and boundary conditions, this hyperbolic PDE can be solved exactly by D'Alembert solution, using Fourier transform, or via. separation of variables. Thus, the problem of string vibrations boils down to solving wave equation under appropriate boundary conditions which has been dealt very elegantly by mathematicians over the years. The real complication arises in situations where either the transverse motions are large enough that the assumption of small vibrations no longer holds or the supports are not ideal giving rise to non-trivial boundary conditions. Under these situations, we can not get exact solutions to the string vibrations problem. Interestingly, real practical applications of string vibrations often take us into this realm.

a e-mail: harkirat@iitk.ac.in
String vibrations are inevitable when we discuss about musical instruments. Indian stringed musical instruments have been subjected to investigation in the works of C.V. Raman[1] and B.C. Deva[2]. The peculiar feature of the Indian stringed musical instruments is the presence of a finite-sized curved bridge. The string wraps and unwraps around the curved bridge, therefore changing the effective length of the vibrating string. The effect of curved bridge is prominent in determining the tonal quality of the instrument. Theoretically, the problem of vibration of string against a rigid wall was mathematically formulated by Amerio[3]. Besides this, sundry theoretical studies have been reported in the literature[4-6]. However, all these studies have been restricted to planar motions of vibrating string. In the present paper, we have studied the non-planar motions of string vibrating against a smooth unilateral obstacle. We have further explored the effect of the obstacle on the dynamics of the string. We have developed a mathematical model taking into consideration the holonomic constraint and derive the governing equation using Hamilton's principle. In addition to this, we analyze the dynamics of a string, explore the possibility of different types of motion and numerically investigate the results.

\section{Mathematical Model}

A schematic representation of the physical system under consideration is shown in figure 1. It comprises one ideal(no bending stiffness) string which vibrates against a smooth obstacle at one of the ends. The string starts from $O$ which is the origin of our system and it is tied to the support at other end which is at distance $L$. The obstacle is assumed to be rigid and fixed in our model. The mathematical form of the 


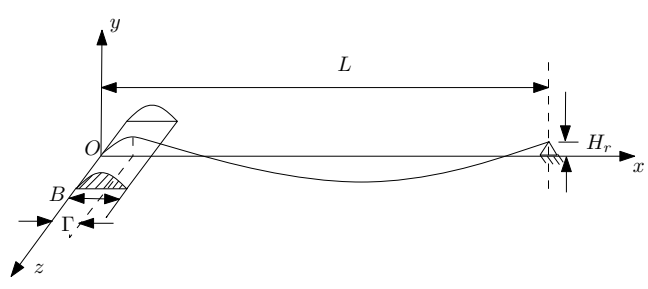

Fig. 1. Schematic representation of the system under consideration

bridge is given by

$$
Y_{B}(x)=A_{P} x(B-x),
$$

where $A_{P}$ is a constant (related to the curvature of the bridge), and $B$ is the length of the bridge. The shape of the obstacle is assumed such that it is curved only along one direction and the cross section in the plane parallel to the string is parabolic. The string has uniform density $\rho$ per unit length, however, the tension varies along the length due to stretching of the string. We further assume that the string wraps perfectly around the obstacle, and thus, remains tangent to the obstacle at the point of separation. The Lagrangian of the system can be written as

$\int_{0}^{L}\left(\frac{1}{2} \rho\left(y_{t}^{2}+z_{t}^{2}\right)-\frac{1}{2} T_{0}\left(y_{x}^{2}+z_{x}^{2}\right)+\frac{E A}{8}\left(y_{x}^{2}+z_{x}^{2}\right)^{2}\right) d x$.

The wrapped portion of the string is constrained to move on the surface of the obstacle. Now, introducing the non-dimensional parameters as

$$
\begin{aligned}
& \bar{x}=\frac{x}{L}, \bar{y}(\bar{x}, \bar{\tau})=\frac{y(x, t)}{h}, \bar{z}(\bar{x}, \bar{\tau})=\frac{z(x, t)}{h}, \gamma=\frac{\Gamma(t)}{L}, \\
& b=\frac{B}{L}, a=A_{p} \frac{L^{2}}{h}, \tau=t \sqrt{\frac{T}{\rho L^{2}}}, \sigma=\frac{E A h^{2} L^{2}}{2 L^{2} T_{0}},
\end{aligned}
$$

where $h$ is the height of the bridge $\left(A_{p} B^{2} / 4\right)$ and $\sigma$ is the non-dimensional stretching nonlinearity parameter of the system. Using the Hamilton's principle, the governing equations for the system can be written as

$$
\bar{y}(\bar{x}, \tau)=a \bar{x}(b-\bar{x}) \quad 0 \leq x \leq \gamma^{-},
$$

and for $\gamma^{+} \leq x \leq L$,

$$
-\frac{\partial^{2} \bar{y}(\bar{x}, \tau)}{\partial \tau^{2}}+\frac{\partial^{2} \bar{y}(\bar{x}, \tau)}{\partial \bar{x}^{2}}+\sigma \frac{\partial}{\partial \bar{x}} \bar{y}_{\bar{x}}\left(\bar{y}_{\bar{x}}^{2}+\bar{z}_{\bar{x}}^{2}\right)=0 .(6)
$$

Since, there is no constraint in the $z$-direction, we can obtain a single PDE for the entire length, i.e, $0 \leq x \leq$ $L$, which is given by

$$
-\frac{\partial^{2} \bar{z}(\bar{x}, \tau)}{\partial \tau^{2}}+\frac{\partial^{2} \bar{z}(\bar{x}, \tau)}{\partial \bar{x}^{2}}+\sigma \frac{\partial}{\partial \bar{x}} \bar{z}_{\bar{x}}\left(\bar{y}_{\bar{x}}^{2}+\bar{z}_{\bar{x}}^{2}\right)=0 .(7)
$$

The boundary conditions in terms of non-dimensional variables are given as

$\bar{y}(\gamma, \tau)=a \gamma(b-\gamma), \bar{y}(1, \tau)=0, \bar{z}(0, \tau)=0, \bar{z}(1, \tau)=0$,

and

$$
\left.\frac{\partial \bar{y}(\bar{x}, \tau)}{\partial \bar{x}}\right|_{\bar{x}=\gamma}=a(b-2 \gamma)
$$

\section{Model order reduction}

The physical system has been reduced to non-linear coupled partial differential equations. The dynamics of the string can be fully understood by finding solutions of these PDEs. However, there is no standard approach to find the closed form exact solutions. In this section, we use Galerkin projection approach to discretize the system by assuming functional form of the approximating functions which satisfies all the geometrical boundary conditions. Moreover, in order to write the expressions succinctly, we will write $x$ instead of $\bar{x}, t$ in place of $\tau$ and drop the explicit dependence of $\mathrm{y}$ on the variables $x$ and $\tau$. Restricting our analysis to one mode, the assumed functional form for $y$-direction motion is given by

$$
\begin{gathered}
y=\frac{a \gamma(b-\gamma)}{1-\gamma}(1-x)+\beta(t) \sin \left(\frac{\pi(x-\gamma)}{1-\gamma}\right) \\
\text { for } \gamma_{1}^{+} \leq x \leq 1,
\end{gathered}
$$

and for $z$-direction motion, we assume

$$
z=\alpha(t) \sin (\pi x) \quad \text { for } 0 \leq x \leq 1 .
$$

Substituting (10)-(11) in (6), we obtain residual term denotes by $R_{y}$. Again, we substitute (5)-(11) followed by (10)-(11) in (7) to get residual forms $R_{z 1}$ and $R_{z 2}$, respectively. To minimize the error, we use Galerkin projection in the following manner

$$
\begin{aligned}
& \int_{0}^{\gamma_{1}} R_{z 1} \sin (\pi x)+\int_{\gamma_{1}}^{1} R_{z 2} \sin (\pi x)=0 \\
& \int_{\gamma_{1}}^{1} R_{y} \sin \left(\frac{\pi\left(x-\gamma_{1}\right)}{1-\gamma_{1}}\right)=0 .
\end{aligned}
$$

Using the assumed functional forms, slope continuity conditions at the point of contact given by

$$
\left.\frac{\partial y}{\partial x}\right|_{x=\gamma_{-}}=a(b-2 \gamma)
$$

which leads to

$$
\left.\frac{\partial y}{\partial x}\right|_{x=\gamma_{+}}=-\frac{a \gamma(b-\gamma)}{1-\gamma}+\frac{\beta(t) \pi}{1-\gamma}
$$

Equating the above two equations, we get

$$
-a \gamma(b-\gamma)+\beta(t) \pi=a(1-\gamma)(b-2 \gamma),
$$

which can be simplifies as

$$
a \gamma^{2}-2 a \gamma+a b-\pi \beta(t)=0 .
$$

The above quadratic equation in terms of $\gamma$ suggests that there are two roots possible. However the only relevant root is

$$
\gamma=1-\sqrt{1-\left(b-\frac{\pi \beta(t)}{a}\right)}
$$

The above equation holds for all time instants, differentiating leads to

$$
\dot{\gamma}=-\frac{\pi \dot{\beta}(t)}{2 a(1-\gamma)}
$$



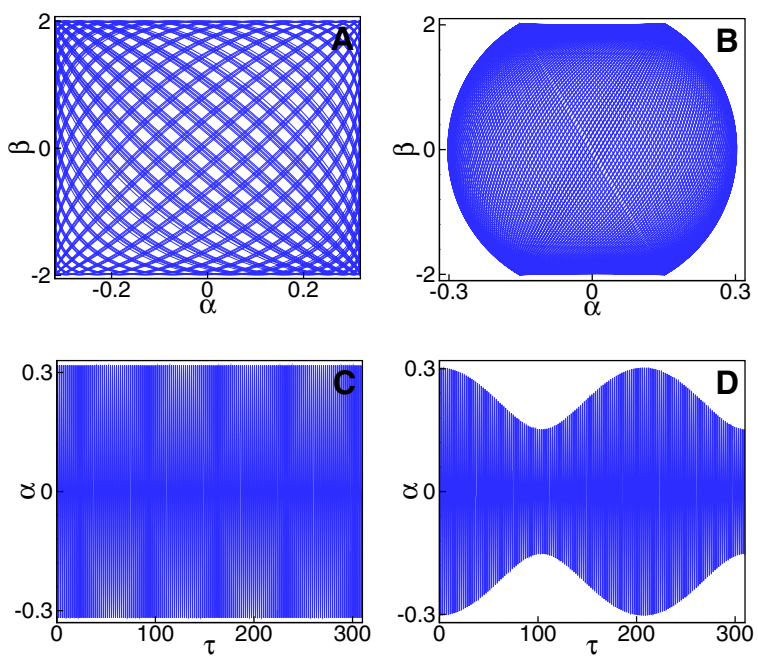

Fig. 2. Representation of stretching nonlinearity. Fig(A) shows the trajectory of a typical point in the absence of stretching nonlinearity whereas fig(B) shows the trajectory of the same point in the presence of stretching nonlinearity under same set of initial conditions $(\alpha(0)=0, \dot{\alpha}(0)=1$, $\beta(0)=2$ and $\dot{\beta}(0)=0)$. Fig $(\mathrm{C})$ and fig(D) shows the $\alpha$ variation corresponding to fig(A) and fig(B), respectively.

and

$$
\ddot{\gamma}=\frac{\dot{\gamma}^{2}}{1-\gamma}-\frac{\pi \ddot{\beta}(t)}{2 a(1-\gamma)} .
$$

Thus, we have obtained the point of contact in terms of modal functions. These expressions can be used to obtain the ODEs, however, ODEs being lengthy are omitted here.

\section{Numerical Simulations}

In this section, we numerically investigate the ODEs, obtained in section 3 , under different set of initial conditions. The obstacle parameters are pertinent to the bridge of Indian classical stringed instruments. In particular, we choose $b=0.06, \sigma=0.001$ and $a=1111$, such that $\frac{a b^{2}}{4}$ is 1 , for our numerical simulations. Any non-zero initial condition in both $\alpha$ and $\beta$ simultaneously results in non-planar motions. The effect of stretching non-linearity on the dynamics of string have also been observed numerically in figure 2 . It is clear from figure 2 that the modal interaction between the perpendicular modes occur only in the presence of stretching nonlinearity. In addition to this, we observe that nature of trajectory of a typical point on the string slowly transforms as we increase the amplitude of oscillations. We explore the different types of non-planar motions in the subsequent discussion.

\subsection{Oscillating trajectories}

It has been numerically found that for small values of $\beta$, the trajectories are oscillating ellipses with time varying major and minor axis. The exchange of energy between the perpendicular modes is quite significant and its strength increases as we increase the amplitude of vibrations. The trajectory of a point and the variation of $\alpha$ and $\beta$ is shown in figure 3A. These types are also observed when the initial disturbances are given in terms of velocity and displacement in $z$ and $y$ direction, respectively. However, these types of non-planar motions are possible only below certain value of $\beta$.

\subsection{Rotating trajectories}

Numerical simulations also divulge that the larger values of $\beta$ engender rotating elliptical trajectories with varying major and minor axis. Thus, any non-zero initial condition in terms of $\alpha$ and $\beta$ give rise to rotating trajectories above certain value of $\beta$. In this case, the extent of energy exchange is much higher as compared to the previous case. We further observe that the maximum value attains by $\alpha$ variation remains same irrespective of the magnitude of the perturbation. The trajectory of a point and the variation of $\alpha$ and $\beta$ is shown in figure $3 \mathrm{~B}$. This transformation of
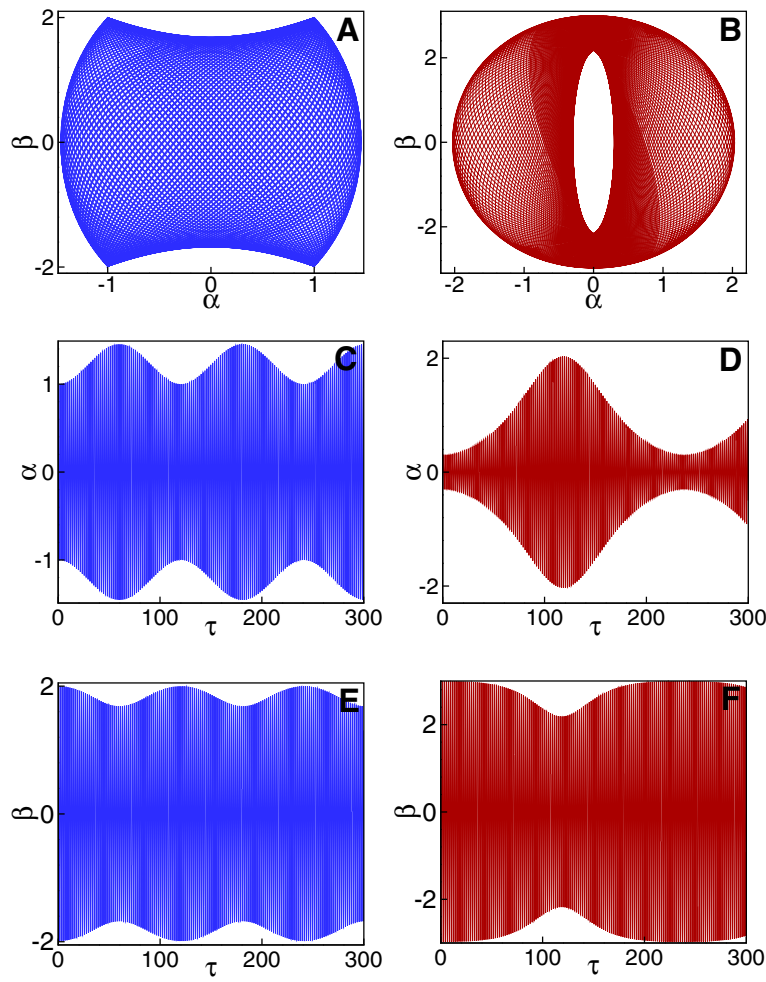

Fig. 3. Some representations of non-planar motions for different set of initial conditions. Fig(A) shows the oscillating elliptical trajectory corresponds to $\alpha(0)=1$ and $\beta(0)=2$, fig $(\mathrm{B})$ shows the rotating elliptical trajectory corresponds to $\dot{\alpha}(0)=1$ and $\beta(0)=3$. Fig $(\mathrm{C}, \mathrm{E})$ and $\operatorname{fig}(\mathrm{D}, \mathrm{F})$ shows the $\alpha$ and $\beta$ variation with respect to nondimensional time corresponding to $\mathrm{fig}(\mathrm{A})$ and $\mathrm{fig}(\mathrm{B})$, respectively.

trajectories denotes the onset of unstable regime for planar motions. 


\section{Conclusion}

It is evident from our analysis that assumption of planar motion does not capture the holistic dynamics of the string. Moreover, in almost all practical scenarios, the motion of the string is undoubtedly non planar. For example, in stringed musical instrument, the string vibrates erratically in space and amplitude of motion is large enough to include the nonlinear effects due to variable tension. Thus, our model is very relevant to such cases and it aptly captures the underlying physics behind 3-dimensional motions of a string vibrating against a unilateral obstacle. It has been found that the oscillating elliptical trajectories transforms into rotating elliptical trajectories for larger amplitudes. The qualitative change in dynamics of string marks the onset of unstable regime which will be analyzed in detail in our future study. Moreover, the obstacle is assumed to be curved only along one direction, taking complex geometry and considering the effect of friction between the string and obstacle will indeed capture the more realistic dynamics.

\section{References}

1. C.V. Raman, On some indian stringed instruments. Proc.Indian Assoc.Cultiv.Sci., 7(1921), 29-33.

2. B.C. Deva, Musical instruments of india: Their history and development. 2nd ed. (Munshiram Manoharlal), New Delhi, India, (1987).

3. L. Amerio. Continuous solutions of the problem of a string vibrating against an obstacle. Rend.Sem.Mat.Univ.Padova, 59:(1978) 67-96.

4. R. Burridge, J. Kappraff, and C. Morshedi. The sitar string, a vibrating string with a one-sided inelastic constraint. SIAM Journal of Applied Mathematics, 42(6):(1982) 1231- 1251.

5. C. P. Vyasarayani, S. Birkett, and J. McPhee. Modeling the dynamics of a vibrating string with a finite distributed unilateral constraint: Application to the sitar. Journal of the Acoustical Society of America, 125(6):(2009) 3673-3682.

6. A.K. Mandal and P. Wahi. Natural frequencies, modeshapes and modal interactions for strings vibrating against an obstacle: Relevance to sitar and veena. Journal of Sound and Vibration, 338:(2015) 42-59. 